\title{
Missing Data Patterns: From Theory to an Application in the Steel Industry
}

\author{
Michal Bechný \\ michal.bechny@scch.at \\ Software Competence Center Hagenberg GmbH \\ Austria \\ Jürgen Zeindl \\ juergen.zeindl@voestalpine.com \\ voestalpine Stahl GmbH
}

Austria

\begin{abstract}
Missing data (MD) is a prevalent problem and can negatively affect the trustworthiness of data analysis. In industrial use cases, faulty sensors or errors during data integration are common causes for systematically missing values. The majority of MD research deals with imputation, i.e., the replacement of missing values with "best guesses". Most imputation methods require missing values to occur independently, which is rarely the case in industry. Thus, it is necessary to identify missing data patterns (i.e., systematically missing values) prior to imputation (1) to understand the cause of the missingness, (2) to gain deeper insight into the data, and (3) to choose the proper imputation technique. However, in literature, there is a wide varity of MD patterns without a common formalization. In this paper, we introduce the first formal definition of MD patterns. Building on this theory, we developed a systematic approach on how to automatically detect MD patterns in industrial data. The approach has been developed in cooperation with voestalpine Stahl $\mathrm{GmbH}$, where we applied it to real-world data from the steel industry and demonstrated its efficacy with a simulation study.
\end{abstract}

\section{CCS CONCEPTS}

- Information systems $\rightarrow$ Data cleaning; - Mathematics of computing $\rightarrow$ Multivariate statistics.

\section{KEYWORDS}

Missing Data, Data Quality, Pattern Detection, Steel Industry.

\section{ACM Reference Format:}

Michal Bechný, Florian Sobieczky, Jürgen Zeindl, and Lisa Ehrlinger. 2021. Missing Data Patterns: From Theory to an Application in the Steel Industry. In 33rd International Conference on Scientific and Statistical Database Management (SSDBM 2021), fuly 6-7, 2021, Tampa, FL, USA. ACM, New York, NY, USA, 6 pages. https://doi.org/10.1145/3468791.3468841

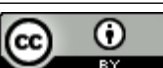

This work is licensed under a Creative Commons Attribution International 4.0 License.

SSDBM 2021, July 6-7, 2021, Tampa, FL, USA

(c) 2021 Copyright held by the owner/author(s).

ACM ISBN 978-1-4503-8413-1/21/07.

https://doi.org/10.1145/3468791.3468841

\author{
Florian Sobieczky \\ florian.sobieczky@scch.at \\ Software Competence Center Hagenberg GmbH \\ Austria \\ Lisa Ehrlinger \\ lisa.ehrlinger@scch.at \\ Software Competence Center Hagenberg GmbH \\ Johannes Kepler University Linz \\ Austria
}

\section{INTRODUCTION}

Reasons for MD are manifold: data integration from multiple sources, where not each attribute is populated by every source, incompletely gathered data in surveys, or technical issues like sensor breakdowns in industrial applications [4, 7]. Since data disappears seldomly in a random fashion, MD can introduce serious bias into data analytics and it is thus crucial to detect and handle MD patterns appropriately. The majority of existing MD research comes from the social sciences and deals with survey data with a focus on the imputation of missing values (cf. [17, 18]). And although, most literature in this area mention "MD patterns" as important concept, there is little work that explicitly focuses on the detection of such patterns. In addition, we found that the notation and patterns proposed are very diverse and that there is no common formalization of MD patterns. The contribution of this paper can be summarized as follows:

(1) To the best of our knowledge, we introduce the first formal definition of MD patterns.

(2) We propose a novel approach based on association analysis and biclustering to reliably detect those MD patterns. The efficacy of the approach was underlined with real-world industrial data from the steel industry.

Section 2 of this paper covers the state-of-the-art on MD pattern. In Section 3, we present the first formal definition of MD patterns, which was a prerequisite for the systematic approach in Section 4, which is applied and evaluated in Section 5.

\section{MD PATTERNS - RELATED WORK}

An observed data set $\mathrm{D}^{n \times m}$ consists of $n$ rows (observations) and $m$ columns (variables). Unless mentioned otherwise, we assume rows to be independent and identically distributed (i.i.d.). The missingness of values in $\mathbf{D}$ is identified by binary matrix $\mathbf{M}^{n \times m}$, where $m_{i j}=1$ if $d_{i j}$ is missing, and $m_{i j}=0$ otherwise. The index $i$ refers to rows from the row set $\mathcal{I}$, and the indices $j$ and $l$ refer to columns from the column set $\mathcal{J}$. The $\mathrm{D}$ can be partitioned into $\mathrm{D}=(\mathrm{X}, \mathrm{Y})$, where $\mathrm{X}^{n \times l}=\left(\mathrm{X}_{1}, \ldots, \mathrm{X}_{l}\right)$ is a set of fully observed variables and $\mathrm{Y}^{n \times k}=\left(\mathrm{Y}_{1}, \ldots, \mathrm{Y}_{k}\right)$ a set of variables containing $\mathrm{MD}, m=l+k$. The $\mathbf{M}$ can be partitioned accordingly, $\mathbf{M}=\left(\mathbf{M}^{\mathrm{X}}, \mathbf{M}^{\mathrm{Y}}\right)$, with zero-valued $\mathbf{M}^{\mathbf{X}}$ and $\mathbf{M}^{\mathbf{Y}}$, whose columns refer to Bernoulli processes (cf. [3]).

There is no standardized list or agreement on the meaning of MD patterns in literature (cf. [1, 5, 13, 17, 20]). Van Buuren [22] 
introduces a MD pattern briefly as $\mathbf{M}$ without any further explanation. Enders [8] describes a MD pattern as "a configuration of observed and missing values in a data set". Independently of the author (cf. [8, 9, 22]), at least an agreement that the MD patterns should be checked and considered, holds for all. In the following, we list MD patterns that commonly appear in related work as well as three patterns that are of specific interest for industrial data. The individual patterns are illustrated in Figure 1.

\section{Common MD patterns:}

- Univariate and multivariate - missingness of the same observations in one or more variables,

- Latent variable - missingness of the entire variable $\xi$, existence of which is only assumed [17],

- Monotone - if $Y$ can be ordered such that if $Y_{j}$ is missing then all $Y_{k}$ with $k>j$ are also missing [17],

- Planned - recording of all variables jointly is impossible, too expensive or causes burden [8],

- General - default case in practice, typically more MD patterns combined with non-systematically MD (cf. $[1,5,20]$ ).

Industry-Specific MD patterns:

- File matching pattern (FMP) is introduced in [17] as case when two variables are never observed jointly. We argue that FMP is of special importance nowadays, when data is queried from multiple information systems. The FMP is typically caused by joining data from heterogeneous sources with different column dimensions, which yields blocks of MD over several variables that can be viewed as a generalization of FMP introduced in [17].

- Line pattern $(L P)$ is caused by a breakdown of multiple sensors and after suitable rearrangements of columns seen as a "line" of MD in $\boldsymbol{M}$ (cf. [7, 13]). Based on our experience from steel industry, we further distinguish between three LP subtypes: perfect, noisy, and asymmetric line pattern (see [6] for details).

- Multi-rate pattern (MRP) - caused by intentional measurement of certain variables less frequently [13].

To avoid excessive length of this paper, we provide a detailed list of MD patterns on our website [6], where we also discuss the differences of MD patterns from Figure 1 with respect to the amount, and cause of MD. The fact that several patterns might have a similar appearance after aligning the rows illustrates the inconsistency in MD research due to the absence of a common formalization.

\section{A FORMALIZATION OF MD PATTERNS}

In this section, we propose the first formal definition of MD patterns. The formalization considers the mathematical properties minimality, completeness, and symmetry.

The more general term pattern is described on Wikipedia as "a regularity in the world ...", and that "the elements of a pattern repeat in a predictable manner" [24]. Thus, loosely speaking, a MD pattern can be seen as a significant violation of the independence structure within $\mathbf{M}^{\mathrm{Y}}$ in either row- or column-wise way. For example, if some set of Y-variables tend to be missing jointly (cf. Figure 1(b,d,h)), we state that related variables form a MD pattern of a positive association. Similarly, if the missingness in one variable can be inferred from the completeness of another variable (cf. Figure 1(e)),
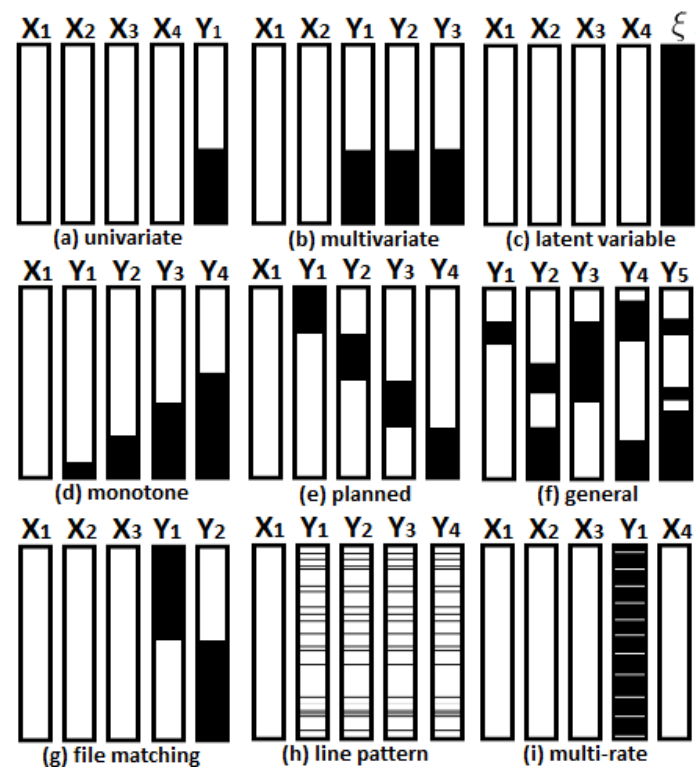

Figure 1: Missing data patterns

then these variables form a MD pattern of a negative association. To investigate MD patterns, we argue that it is sufficient to focus on $\mathrm{M}^{\mathrm{Y}}$ only, since it captures all relevant information.

Definition 3.1. [Global MD pattern] A p-dimensional global MD pattern of a $k$-dimensional array of i.i.d. Bernoulli processes is a subset $J \subseteq\{1, \ldots, k\},|J|=p$, such that:

$$
\forall_{j \in J} \exists_{l \in J \backslash\{j\}} \quad P\left[\mathbf{M}_{\cdot j}=1, \mathbf{M}_{\cdot l}=1\right] \neq P\left[\mathbf{M}_{\cdot j}=1\right] P\left[\mathbf{M}_{\cdot l}=1\right] .
$$

The statement to the right of the quantifiers is denoted as $\mathcal{D}(\cdot, j, l)$ and interpreted as a significant violation of independence between events of MD for $\mathrm{Y}_{j}$ and $\mathrm{Y}_{l}$. This implies that every variable "in $J$ " has at least one "partner" with which it is significantly dependent or associated in terms of $\mathbf{M}$. To emphasize that the MD pattern is related to a subset of variables $J$, we call it (global) J-pattern.

Definition 3.2. [Completeness]

Let $L \subset\{1 \ldots k\} \backslash J$. A p-dimensional J-pattern of the $k$-dimensional array of i.i.d. Bernoulli processes is complete iff

$$
\forall_{j \in J} \quad \forall_{l \in L} \neg \mathcal{D}(\cdot, j, l) .
$$

The completeness of a $J$-pattern implies that there is no further dependency outside of the subset $J$.

Definition 3.3. [Minimality] Ap-dimensional J-pattern of the k-dimensional array of i.i.d. Bernoulli processes is minimal iff

$$
\forall_{L: L \subsetneq J \neq \emptyset} \quad \exists_{j \in J \backslash L} \quad \exists_{l \in L} \quad \mathcal{D}(\cdot, j, l) .
$$

A minimal $J$-pattern refers to a set of associated variables that cannot be split into two complete "sub-patterns" $L$ and $J \backslash L$.

Definition 3.4. [Symmetry]

A p-dimensional J-pattern of the $k$-dimensional array of i.i.d. Bernoulli processes is symmetric iff

$$
\forall_{j \in J} \forall_{l \in J \backslash\{j\}} P\left(\mathbf{M}_{\cdot j}=1 \mid \mathbf{M}_{\cdot l}=1\right)=P\left(\mathbf{M}_{\cdot l}=1 \mid \mathbf{M}_{\cdot j}=1\right) .
$$


A $J$-pattern is symmetric if the "one-directional" relationships $P\left[\mathbf{M}_{\cdot j}=1 \mid \mathbf{M}_{\cdot l}=1\right]$ and $P\left[\mathbf{M}_{\cdot l}=1 \mid \mathbf{M}_{\cdot j}=1\right]$ are of the same strength for all pairs of variables within the set $J$.

In industrial applications, observations within columns of $\mathrm{M}^{\mathrm{Y}}$ are rarely i.i.d., because the data set is usually a result of a database query joining multiple data sources. These data sources can have different probability of containing MD. If $P\left(\mathbf{M}_{\cdot j}=1\right)$ varies across observations, it is more realistic to consider columns of $\mathrm{M}^{\mathrm{Y}}$ as samples from "non-stationary Bernoulli processes":

$$
\begin{aligned}
m_{i j}: \Omega & \rightarrow\{0,1\} \\
P\left(m_{i j}=1\right) & =p_{i j},
\end{aligned}
$$

where $p_{i j}$ highlights the dependency of obtaining MD on the row $i$.

DeFINITION 3.5. [Non-stationary MD pattern] Let $I \subset\{1, \ldots, n\}$ and $J \subseteq\{1 \ldots k\}$, where $|J|=p$. Further, let

$$
\mathcal{D}(i, j, l):=P\left[m_{i j}=1, m_{i l}=1\right] \neq P\left[m_{i j}=1\right] P\left[m_{i l}=1\right] .
$$

The subset of variables $J$ considered on the subset of observations $I$ is called a $p$-dimensional non-stationary MD pattern of a $k$-dimensional array of Bernoulli processes iff

$$
\forall_{i \in I} \forall_{j \in J} \exists_{l \in J \backslash\{j\}} \mathcal{D}(i, j, l) .
$$

A non-stationary MD pattern is an extension of a $J$-pattern identified on a subset of rows I for which Def. 3.1 holds. It can be alternatively denoted as a local f-pattern or local MD pattern.

The identification of local patterns is more complex, since it requires to evaluate associations jointly for both dimensions of $\mathrm{M}^{\mathrm{Y}}$. The knowledge about $I$ for which a certain dependency/association was found, can help to select a more appropriate action (e.g., deletion, type of imputation) than dealing with all MD in a given variable at the same way.

\section{APPROACH TO DETECT MD PATTERNS}

Traditional strategies for detecting MD patterns are often not appropriate for data of higher dimensionality, which is a rule rather than an exception in industry. Examples are the visual inspection of an aggregation plot of $\mathrm{M}^{\mathrm{Y}}$ or the manual verification of different combinations between missing and observed components of the data [17, 22]. Investigation of $\mathbf{M}^{\mathrm{Y}}$ with "only" 20 variables can yield up to $2^{20}$ combinations, making such strategies hardly feasible.

Therefore, we propose a new approach to automate detection of any MD pattern, with a particular focus on patterns appearing in industrial applications. The core idea is to discover significant regularities in the structure of $\mathrm{M}^{\mathrm{Y}}$. The four iterative steps of our approach are illustrated in Figure 2:

Step (1): D is uniquely transformed to $\mathrm{M}=\left(\mathrm{M}^{\mathrm{X}}, \mathrm{M}^{\mathrm{Y}}\right)$ using knowledge about the encoding of MD.

Step (2): To detect global MD patterns, associations and dependencies among $\mathbf{M}^{\mathrm{Y}}$ columns are investigated. Significant relations found are used as an input for hierarchical clustering or a dependency graph to effectively identify subset(s) of variables in accordance with Def. 3.1. An overview on the measures with their properties is given in Figure 2 and Section 4.1, and further details in [6]. Step (3): To detect local MD patterns, two biclustering methods are introduced in Section 4.2: Ensemble Biclustering using iterative Binary Biclustering of Gene sets $(\mathrm{EB}+\mathrm{iBBiG})[12]$ and the Latent Block

\begin{tabular}{|c|c|c|c|}
\hline Method & Discovery & $\begin{array}{l}\text { Significance } \\
\text { evaluation }\end{array}$ & $\begin{array}{l}\text { Similarity } \\
\text { measure }\end{array}$ \\
\hline$\chi^{2}$ & Dependencies & Test & No \\
\hline$\Phi$ & $\begin{array}{l}\text { Dependencies, positive \& } \\
\text { negative associations }\end{array}$ & Test & No \\
\hline 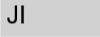 & Pos. associations & Simulation & Yes \\
\hline $\mathrm{KI}$ & Positive associations & Simulation & Yes \\
\hline DI & Asymmetric associations & Simulation & No \\
\hline \multicolumn{4}{|c|}{$\sqrt{7}$} \\
\hline \multicolumn{4}{|c|}{ Hierarchical Clustering / dependency graph } \\
\hline & & meter setting & \\
\hline
\end{tabular}

(1) Data Transformation

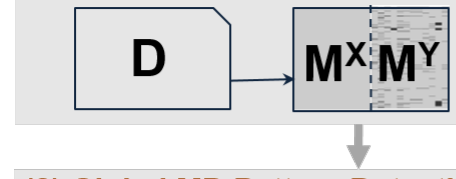

(2) Global MD Pattern Detection

(3) Local MD Pattern Detection

\begin{tabular}{ll}
\hline Method & MD Patterns \\
\hline Ensemble Biclustering (EB+iBBiG) & LP, FMP, multi-rate \\
Latent Block Model (LBM) & Any local MD pattern \\
\hline &
\end{tabular}

(4) Reporting, Refinement, Post-Processing

Figure 2: Approach to detect MD patterns in steel industry

Model (LBM) [10]. Biclustering finds associations simultaneously in rows and columns of $\mathbf{M}^{\mathrm{Y}}$, which makes it suitable to detect local MD patterns in accordance with to Def. 3.5. The results from step (2) can be used to select proper setting for both biclustering methods. Step (4): The reported results are used to refine the data set, to reformulate database queries, or to impute MD.

While the entire approach is presented in Figure 2, the focus in this paper is on step (2) and (3). Step (1) is implemented according to the requirements for voestalpine Stahl $\mathrm{GmbH}$ (details in Section 5.2.1) and step (4) is non-trivial and therefore planned as follow-up research to this work.

\subsection{Global MD Pattern Detection}

To identify global MD patterns according to Def. 3.1 and step (2), the dependencies and associations among the $\mathrm{M}^{\mathrm{Y}}$ variables need to be evaluated using the measures from the step (2) from Figure 2. Suitability of individual methods to detect various MD patterns, their relation to properties (completeness from Def. 3.2, minimality from Def. 3.3, and symmetry from Def. 3.4), as well as details on their functional form and evaluation of statistical significance are discussed in detail in the online supplementals to this paper [6].

Based on the similarity or dependency matrix storing all pairwise comparisons of $\mathrm{M}^{\mathrm{Y}}$ variables, we suggest to construct a dendrogram using hierarchical clustering or to visualize the respective matrix as a graph. By using an appropriate threshold, both procedures can automatically detect subsets of the related variables $J$ (cf. Def. 3.1). The choice of the threshold depends on the strength of the associations that the detected paterns are expected to have. 


\subsection{Local MD Pattern Detection}

In this section, we discuss two biclustering methods for detection of local MD patterns (cf. Def. 3.5) and step (3) in Figure 2. Biclustering is an unsupervised method simultaneously grouping rows and columns of a data set [14]. A bicluster $\mathbf{B}(I, J)$ is a submatrix of data identified by a subset of rows, $I \subseteq \mathcal{I}$ satisfying certain similarity constrains on a subset of columns $J \subseteq \mathcal{J}$. In our application, we found biclustering highly effective to identify data cells $d_{i j}$ missing due to either a LP or a FMP (see Section 5). The parts of $\mathbf{M}^{\mathrm{Y}}$ affected by a FMP typically built a perfect bicluster consisting of only 1 's. The biclusters corresponding to a LP are dense, depending on the type of the LP (cf. [6]). Both FMP and LP yield biclusters with a strong positive association between both sets $I$ and $J$. The biclusters consisting of mostly 0's are of little importance for MD pattern detection, since they correspond to the observed part of the data.

In our application, we compared and evaluated the following two biclustering techniques: $E B+i B B i G$ and $L B M$. The iBBiG[12] algorithm is suitable for the fast extraction of dense biclusters from a sparse binary matrix, which makes it particularly useful for the detection of positively associated local MD patterns. We applied $\mathrm{iBBiG}$ within the framework of Ensemble Biclustering (EB) to increase the robustness of the results. Second, we evaluated the LBM - which aims to identify the distribution of $\mathrm{M}^{\mathrm{Y}}$ - and enables to detect arbitrary type of MD pattern.

4.2.1 Ensemble Biclustering using iBBiG. The $\mathrm{iBBiG}$ was proposed as a meta-analytic tool for discovering associations in sparse binarized genetical data sets [12]. It has several features that make it suitable for our work: it extracts dense biclusters from data, it allows presence of the 0 -valued noise within biclusters, and it allows biclusters' overlap [12]. The iBBiG is a heuristic based on genetic algorithm that iterates over 3 main steps: (i) bicluster fitness score, (ii) heuristic search, and (iii) iterative extraction [12], which we describe in detail in [6]. Since iBBiG terminates when no more bicluster can be found, it is suggested to specify an overestimate for the expected number of biclusters in the data [12]. In our application, we suggest to use the number of global MD patterns identified in step (2). Output of the $\mathrm{iBBiG}$ is a set of dense biclusters, that identify rows and columns of $\mathrm{M}^{\mathrm{Y}}$ positively associated in terms of the missingness in $\mathrm{Y}$ (cf. Def. 3.5). To increase the robustness of the final solution, we apply the iBBiG within the framework of EB [21]. The core idea of $\mathrm{EB}$ is to run the desired biclustering algorithm multiple times under different starting seeds, and to identify the final robust biclustering solution as an intersection of the individual biclusters satisfying certain similarity criteria [16]. More details are provided in [6].

4.2.2 Latent Block Model. The objective of LBM is to identify the underlying distribution of $\mathrm{M}^{\mathrm{Y}}$ by applying a mixture model jointly on its rows and columns [10]. The LBM assumes partitioning of $I$ and $J$ into $Q$-row and $L$-column classes that jointly express a block-structure of $\mathrm{M}^{\mathrm{Y}}$ and thus, it compress information about its distribution. Therefore, the LBM is highly suited to our setting, since knowledge of the $\mathrm{M}^{\mathrm{Y}}$ distribution is key for MD pattern detection. As a result, $\mathrm{M}^{\mathrm{Y}}$ can be segmented into $Q \times L$ exclusive homogeneous blocks (= biclusters), enabling identification of arbitrary local MD pattern (cf. Def. 3.5). For example, dense blocks can be used to detect rows and columns affected by multivariate pattern, FMP or different types of LP. Further, blocks consisting of (mostly) 0's can be interpreted as parts of complete data which are unlikely to form any local MD pattern. In addition, the analysis of dependencies between individual column classes can be used to describe global MD patterns, which makes LBM universally applicable. Based on the number of global MD patterns found, we suggest to specify the grid for $Q, L$, and then to choose the optimal combination based on the Bayesian information criterion (BIC) (cf. [15]). For details, we refer to $[2,11,19]$ and our supplemental material [6].

\section{EVALUATIONS}

This section provides an evaluation to detect local MD patterns related to LP and FMP from data of voestalpine Stahl GmbH.

\subsection{Application Scenario with Steel Data}

Table 1 show a summary of 3 data sets containing sensor measurements from the steel mill that were provided for our research. The largest of the data sets (DS1) was provided to us primarily for the development of our approach and testing its feasibility on big data. The causes and the patterns of missingness in DS1 were unknown. To enable quantifying and testing of the detection capacity of our approach, voestalpine Stahl GmbH simulated several LPs and FMPs of different sizes containing $0-80 \%$ of 0 -valued noise within $\mathrm{M}^{\mathrm{Y}}$ of DS2 and DS3. To evaluate robustness, both DS2 and DS3 further contained $5 \%$ of randomly MD that didn't follow any pattern. For a clear presentation of the results, the names of variables following some MD patterns begin with prefix " $x$ " whereas the noisy variables begin with " $n$ ". For both DS2 and DS3, the description of the true MD patterns was provided to us post hoc in a form of a list of variable groups forming global patterns, and within reference matrices $\mathbf{M}_{\text {ref }}^{\mathbf{Y}} 1$ storing information about local patterns. Detection of global MD patterns was evaluated by comparison of subsets $J$ s found in step (2) with the reference list, and the local MD patterns from step (3) were evaluated by comparison of $\mathbf{M}_{\text {pred }}^{\mathrm{Y}}{ }^{2}$ with $\mathbf{M}_{\text {ref }}^{\mathrm{Y}}$.

Table 1: Evaluation data sets. Sim. =Simulated, $k=$ number of $\mathrm{M}^{\mathrm{Y}}$ columns, Noise = amount of unsystematic MD added.

\begin{tabular}{|l|l|l|l|l|l|l|}
\hline DS & Rows & Columns & $k$ & Sim. & Patterns & Noise \\
\hline DS1 & 193,700 & 557 & 22 & No & Unknown & $0 \%$ \\
\hline DS2 & 2,641 & 1,125 & 73 & Yes & 3 FMP, 4 LP & $5 \%$ \\
\hline DS3 & 2,500 & 1,000 & 108 & Yes & 18 LP & $5 \%$ \\
\hline
\end{tabular}

\subsection{Experiments and Evaluations}

The analysis of DS1, DS2, and DS3 followed the steps from Figure 2.

5.2.1 Data Transformation. For step (1), $\mathrm{M}^{\mathrm{Y}}$ was obtained using knowledge about the encoding of MD in each dataset (-99, or NA). Although existing metrics to measure MD are often restricted to the detection of null values, in practice, also default values such as "01/01/2000" or "NaN" values need to be considered.

\footnotetext{
${ }^{1}$ The cell (i,j) of $\mathbf{M}_{\text {ref }}^{\mathrm{Y}}$ equals 1, if the corresponding cell of $\mathbf{M}^{\mathrm{Y}}$ belongs to some of the LP/FMP simulated, and equals 0 otherwise

${ }^{2}$ The cell $(\mathrm{i}, \mathrm{j})$ of $\mathbf{M}_{\text {pred }}^{\mathrm{Y}}$ it equals 1 , if the corresponding cell of $\mathrm{M}^{\mathrm{Y}}$ was detected as some LP/FMP, and equals 0 otherwise
} 
5.2.2 Detection of global MD patterns. Since no specific patterns were expected for DS1, we evaluated the correlation, as the most widely applicable measure from Section 4.1. The only significant value of $\Phi=0.35$ was found for $\{\mathrm{x} 224, \mathrm{x} 245\}$. Next, several further significant global patterns were found based on KI, which similarly to $\Phi>0$ correspond to a co-occuring MD.

For DS2 and DS3, we evaluated KI and DI since both measures are suitable to detect positively associated $\mathrm{M}^{\mathrm{Y}}$ variables, which were expected in these data sets. Based on the significant associations we applied hierarchical clustering and/or constructed graphs (see Figure 3), from which we correctly detected all 25/25 global MD patterns ( 7 for DS2 and 18 for DS3). Another desirable result was that our approach assigned only signal variables " $x$ " and none of the noisy variables " $n$ " to global MD patterns.

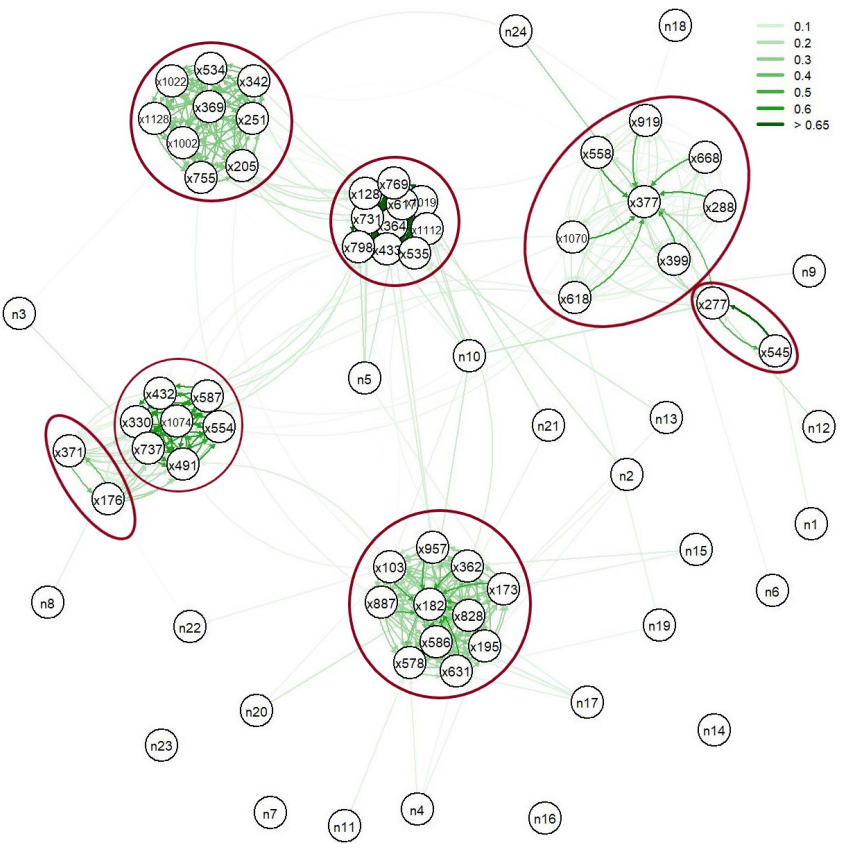

Figure 3: Global MD patterns detected in DS2 based on DI.

5.2.3 Detection of local MD patterns. Based on the number of global MD patterns detected, we set the expected amount of biclusters in the data for $\mathrm{EB}+\mathrm{iBBiG}$, and the grid for $Q, L$ values for which LBM was estimated. Based on the aggregated results from $\mathrm{EB}+\mathrm{iBBiG}, 4,7$, and 18 robust dense biclusters of different sizes and concentrations of 1's were identified from DS1, DS2, and DS3, respectively. In DS1, we identified 4 non-simulated MD patterns affecting 490-935 rows in 2-3 variables, referring to a strong positive local patterns with co-occurring MD. For DS2 and DS3, the biclusters found by $\mathrm{EB}+\mathrm{iBBiG}$ grouped all variables correctly with respect to $M D$ patterns simulated, and detected always at least a part of affected rows. Another desirable result was that $\mathrm{EB}+\mathrm{iBBiG}$ didn't detect any of the noisy variables as a part of any bicluster.

The LBM for DS1 did not converge for any of the combinations of $Q$ and $L$. A possible reason is the very low incidence of $\mathrm{MD}(\approx$ $2.3 \%$ ), which does not form a homogeneous block structure even though several global MD patterns had been detected. For DS2 and DS3, the optimal block structure $(Q=8, L=10)$ for DS2 (see Figure $4)$, and $(Q=21, L=21)$ for DS3 was identified according to the maximal BIC. Finally, the local MD patterns were found as blocks having the mean $>0.5$. A desirable result was that non of those patterns contained any noisy variable. Further, the grouping of the affected variables was correct, even-though some of the simulated patterns were splitted into several blocks.

For the final comparison of EB+iBBiG and LBM on DS2 and DS3, we compared corresponding $\mathbf{M}_{\text {pred }}^{\mathrm{Y}}$ and $\mathbf{M}_{\text {ref }}^{\mathrm{Y}}$ based on which we calculated $\mathrm{BACC}^{3}, \mathrm{TPR}^{4}$, and $\mathrm{TNR}^{5}$. Table 2 shows that $\mathrm{EB}+\mathrm{iBBiG}$ reached better BACC and TPR than LBM. On the other hand, slightly higher and almost perfect specificity was obtained with LBM.

Table 2: Comparison of biclustering algorithms

\begin{tabular}{|l|l|l|l|l|}
\hline Data & Algorithm & BACC $^{\mathbf{1}}$ & TPR $^{2}$ & TNR $^{\mathbf{3}}$ \\
\hline \multirow{2}{*}{ DS2 } & EB+iBBiG & $81.02 \%$ & $62.25 \%$ & $99.79 \%$ \\
& LBM & $79.02 \%$ & $58.06 \%$ & $99.97 \%$ \\
\hline \multirow{2}{*}{ DS3 } & EB+iBBiG & $91.12 \%$ & $82.31 \%$ & $99.91 \%$ \\
& LBM & $82.03 \%$ & $64.15 \%$ & $99.90 \%$ \\
\hline
\end{tabular}

5.2.4 Discussion of the Experiments. For DS1, we identified several global and local patterns indicating the co-occurrence of MD, which might be valuable information for a joint imputation. We plan to investigate the extent to which the runtime could be reduced for the overall imputation process in future research.

The evaluations with DS2 and DS3 showed that all identified global patterns correctly grouped sets of variables forming the patterns simulated. Another desirable result was that our approach assigned only signal variables " $x$ " and none of the noisy variables " $n$ " to any MD pattern detected. Further, we conclude that biclustering is a suitable technique to detect local MD patterns, since in our application focused on the detection of LPs and FMPs, all for DS3, and all but one for DS2 of the simulated biclusters were detected reliably. The single not completely detected local MD pattern in DS2 corresponded to a LP consisted of $80 \%$ noise and was much larger than the other biclusters, which caused lower TPR on DS2 for both approaches. While the TNR was similar and greater than $99 \%$ in all cases, the $\mathrm{EB}+\mathrm{iBBiG}$ outperformed the LBM in terms of much higher sensitivity ( $81 \%$ vs $79 \%$ for DS2, and $82 \%$ vs. $64 \%$ for DS3). While the execution time of $\mathrm{EB}+\mathrm{iBBiG}$ was in the order of a few minutes for each data set, the estimation of all combinations of $Q$ and $L$ to find the optimal LBM required approx. 10 hours (i.e., 20-30 minutes per combination) per data-set. If suboptimal solutions are okay and a suitable guess of $(Q, L)$ is available (e.g., due to analysis of global MD patterns), the estimation takes only a few minutes.

We conclude, that $\mathrm{EB}+\mathrm{iBBiG}$ is the preferable approach to detect industry-specific MD patterns due to (i) comparable and almost perfect specificity, (ii) much better sensitivity, (iii) short computational time, and (iv) no need for parameter tuning in contrast to LBM.

\footnotetext{
${ }^{3}$ balanced accuracy $=\frac{\text { sensitivity }+ \text { specificity }}{2}$

${ }^{4}$ true positive rate $=$ sensitivity $=\frac{T P}{T P+F N}$

${ }^{5}$ true negative rate $=$ specificity $=\frac{T N}{T N+F P}$
} 


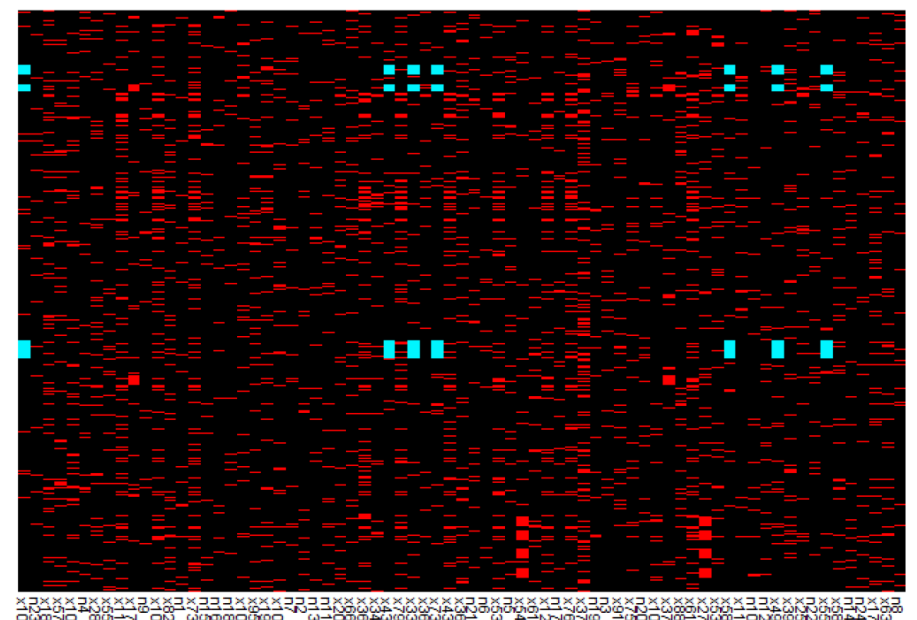

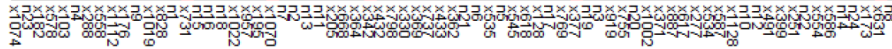

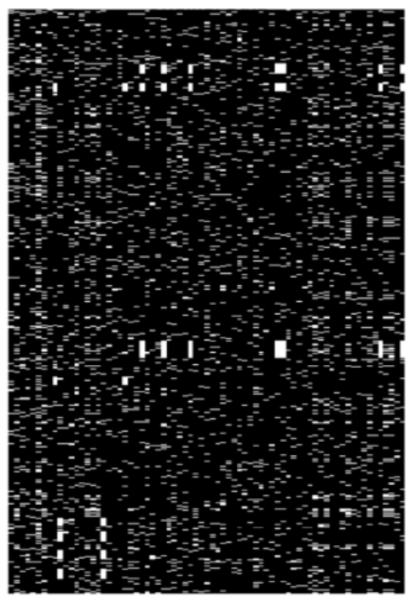

Original data

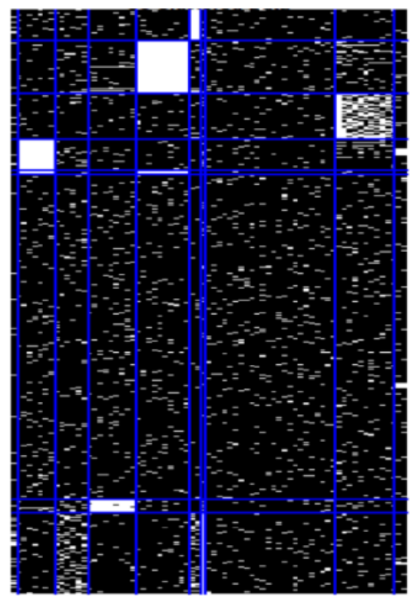

Co-clustered data

Figure 4: Visualization of a sample FMP (in blue) detected in DS2 (left), and the comparison between original and co-clustered $M^{Y}$ data based on the final LBM for DS2 (right).

\section{CONCLUSION}

In this paper, we made a twofold contribution: (1) we introduced the first formal definition for MD patterns, which was used to (2) propose a systematic approach how to detect MD patterns. The approach has been applied to real-world steel data from voestalpine Stahl GmbH. We demonstrate the efficacy of our approach with a simulation study, where $25 / 25$ of global MD patterns could be detected reliably. The evaluations showed that $\mathrm{EB}+\mathrm{iBBiG}$ is specifically suited to detect positively associated local MD patterns (i.e., LPs and FMPs) in a productive environment due to the higher TPR and shorter runtime when compared to LBM. The LBM has the potential to detect other types of MD patterns too, since its results capture the distribution of the missingness matrix.

Several researchers (cf. $[9,23])$ stated without empirical proof that knowledge about MD patterns enhances imputation quality. Other researchers [17, 22] suggested improved imputation procedures for specific MD patterns. As completion of step (4), we are currently investigating the integration of knowledge about MD patterns into the imputation process to increase its accuracy and runtime performance.

\section{ACKNOWLEDGMENTS}

The research in this paper has been funded by BMK, BMDW, and the Province of Upper Austria in the frame of the COMET Programme managed by FFG.

\section{REFERENCES}

[1] Patricia Berglund and Steven G. Heeringa. 2014. Multiple Imputation of Missing Data Using SAS. SAS Institute Inc., Cary, North Carolina, USA.

[2] Parmeet Bhatia, Serge Iovleff, and Gérard Govaert. 2014. Blockcluster: An R Package for model based co-clustering. Fournal of Statistical Software 76, 9 (2014), $1-24$

[3] David Roxbee Cox. 2017. The theory of stochastic processes. Routledge, Abingdonon-Thames, England, UK.

[4] Tamraparni Dasu and Theodore Johnson. 2003. Exploratory Data Mining and Data Cleaning. Vol. 479. John Wiley \& Sons, Hoboken, NJ, USA.

[5] Edith D. de Leeuw, Joop Hox, and Mark Huisman. 2003. Prevention and Treatment of Item Nonresponse. Journal of Official Statistics 19, 2 (2003), 153-176.
[6] Lisa Ehrlinger. 2021. Automated and Continuous Data Quality Measurement. http://dqm.faw.jku.at/

[7] Lisa Ehrlinger, Thomas Grubinger, Bence Gabor Varga, Mario Pichler, Thomas Natschläger, and Jürgen Zeindl. 2018. Treating Missing Data in Industrial Data Analytics. In Proceedings of the 13th International Conference on Digital Information Management (ICDIM). IEEE, Berlin, Germany, 148-155.

[8] Craig K Enders. 2010. Applied Missing Data Analysis. Guilford Press, NY, USA.

[9] Sara Johansson Fernstad. 2019. To Identify What is Not There: A Definition of Missingness Patterns and Evaluation of Missing Value Visualization. Information Visualization 18, 2 (2019), 230-250.

[10] Gerard Govaert and Mohamed Nadif. 2003. Clustering with Block Mixture Models. Pattern Recognition 36 (02 2003), 463-473.

[11] Gérard Govaert and Mohamed Nadif. 2013. Co-Clustering: Models, Algorithms and Applications. John Wiley \& Sons, Inc., New York, NY, USA.

[12] Daniel Gusenleitner, Eleanor A. Howe, Stefan Bentink, John Quackenbush, and Aedín C. Culhane. 2012. iBBiG: iterative binary bi-clustering of gene sets. Bioinformatics 28, 19 (07 2012), 2484-2492.

[13] Syed Imtiaz and Sirish Shah. 2008. Treatment of Missing Values in Process Data Analysis. The Canadian fournal of Chemical Engineering 86, 5 (2008), 838-858.

[14] Adetayo Kasim, Ziv Shkedy, Sebastian Kaiser, Sepp Hochreiter, and Willem Talloen. 2016. Applied Biclustering Methods for Big and High-Dimensional Data using $R$. CRC Press, Boca Raton, Florida.

[15] Christine Keribin, Vincent Brault, Gilles Celeux, and Gérard Govaert. 2015. Estimation and selection for the latent block model on categorical data. Statistics and Computing 25, 6 (2015), 1201-1216.

[16] Tatsiana Khamiakova. 2013. Statistical Methods for Analysis of High Throughput Experiments in Early Drug Development. Ph.D. Dissertation. Hasselt University.

[17] Roderick J. A. Little and Donald B. Rubin. 2002. Statistical Analysis with Missing Data, Second Edition. John Wiley \& Sons, Inc., New York, NY, USA.

[18] Steven F Messner. 1992. Exploring the Consequences of Erratic Data Reporting for Cross-National Research on Homicide. Journal of Quantitative Criminology 8, 2 (1992), 155-173.

[19] Radford M Neal and Geoffrey E Hinton. 1998. A View of the EM Algorithm that Justifies Incremental, Sparse, and Other Variants. In Learning in Graphical Models. Springer, Dordrecht, 355-368.

[20] Joseph L. Schafer and John W. Graham. 2002. Missing Data: Our View of the State of the Art. Psychological Methods 7, 2 (2002), 147-177.

[21] Fan Shi, Christopher Leckie, Geoff MacIntyre, Izhak Haviv, Alex Boussioutas, and Adam Kowalczyk. 2010. A bi-ordering approach to linking gene expression with clinical annotations in gastric cancer. BMC Bioinformatics 11, 1 (2010), 477.

[22] Stef Van Buuren. 2007. Multiple Imputation of Discrete and Continuous Data by Fully Conditional Specification. Statistical Methods in Medical Research 16, 3 (2007), 219-242.

[23] Hai Wang and Shouhong Wang. 2008. Data Mining with Incomplete Data. In Data Warehousing and Mining: Concepts, Methodologies, Tools, and Applications. IGI Global, Hershey, Pennsylvania, 3027-3032.

[24] Wikipedia. 2019. Pattern. Online. https://en.wikipedia.org/wiki/Pattern. 